\title{
Large Coronary Intramural Hematoma Presenting as Acute Coronay Syndrome
}

\author{
Ibenchekroun M,* Kone G, Fellat N \\ Mohamed V University Medical School, Ibn Sina University hospital, National League against cardiovascular disease, Morocco
}

\begin{abstract}
Isolated spontaneous coronary intramural hematoma is a unique subset of spontaneous coronary artery dissection that is characterized by a hemorrhage limited to the medial- adventitial layers, causing subsequent hematoma formation without visible intimal flaps.

It is infrequent and serious coronary vessel wall pathology, with poorly understood underlying pathogenic mechanisms. Affected individuals may present with a broad spectrum of symptoms ranging from acute coronary syndromes (ACS) to cardiogenic shock or even sudden cardiac death. The disease entity causes challenges in terms of both diagnostics and treatment strategy. We report a case of intramural coronary hematoma in a patient presenting with non ST--segment elevation myocardial infarction.
\end{abstract}

Keywords: Intramural hematoma, Pathogenic mechanisms, Lumen compromise, Coronary blood, Clopidogrel

\section{Introduction}

Spontaneous coronary intramural hematoma is a unique and rare subset of spontaneous coronary artery dissection (SCAD), where a hemorrhage within the vessel wall is thought to be the underlying cause, leading to separation of the mural layers. The hematoma formation is limited to the medial-adventitial layers, and no flaps are visible when assessed with tomographic techniques. ${ }^{1,2}$ This serious condition usually presents as acute coronary syndrome (ACS), but symptoms may range from chest pain to cardiogenic shock or sudden death, ${ }^{1,2}$ depending on hematoma location, number of affected vessels, lumen compromise, and restriction of coronary blood flow. It is more frequent in young women, particularly in the peri and post-- partum period and with oral contraceptive use, and in young and middle--aged patients without obvious cardiovascular risk factors. ${ }^{3,4}$ Management strategies may range from conservative medical treatment to percutaneous or surgical interventions depending upon the anatomy, extent of the hematoma and the clinical circumstances. ${ }^{5,6}$

\section{Case Report}

A 64--year old female was admitted due to sudden onset of chest pain. Cardiovascularrisk factors included hypertension. The ECG showed inverted T waves in inferior and low lateral leads and Troponin--I was elevated $(8,52 \mathrm{ng} / \mathrm{ml})$. The patient was treated with a non--STEMI medical treatmentincluding aspirin, clopidogrel and low-- molecular weight heparin. The echocardiogram disclosed normal wall motions and preserved EF. A subacute coronary angiography showed an ectasic right coronary artery, where we detected a large intra mural hematoma starting from mid segment of the RCA. Unfortunately, neather the IVUS or the OCT is available in our hospital. We performed a coronary computed tomography for a more detailed evaluation of the morphological features of the vessel wall. We therefore decided to continue conservative treatment and intensified the medical therapy. She was discharged after one week in a stable clinical condition. A repeat angiogram three months later showed an improvement in the intra muralhematoma (Figures 1-4).

\begin{tabular}{|l|l|}
\hline \hline Quick Response Code: & *Corresponding author: Ibenchekroun M, Mohamed V University Medical School, Ibn Sina \\
University hospital, National League against cardiovascular disease, Morocco \\
Received: 21 March, 2021 \\
Citation: Ibenchekroun M, Kone G, Fellat N. Large Coronary Intramural Hematoma Presenting \\
CR.2021.01.000501
\end{tabular}




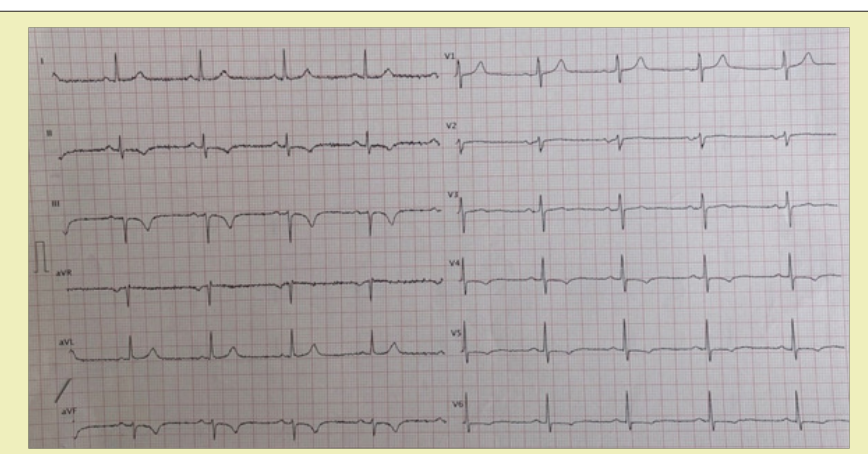

Figure 1: An electrocardiogram showed inverted T waves in leads II, III, aVf and V4 to V6.
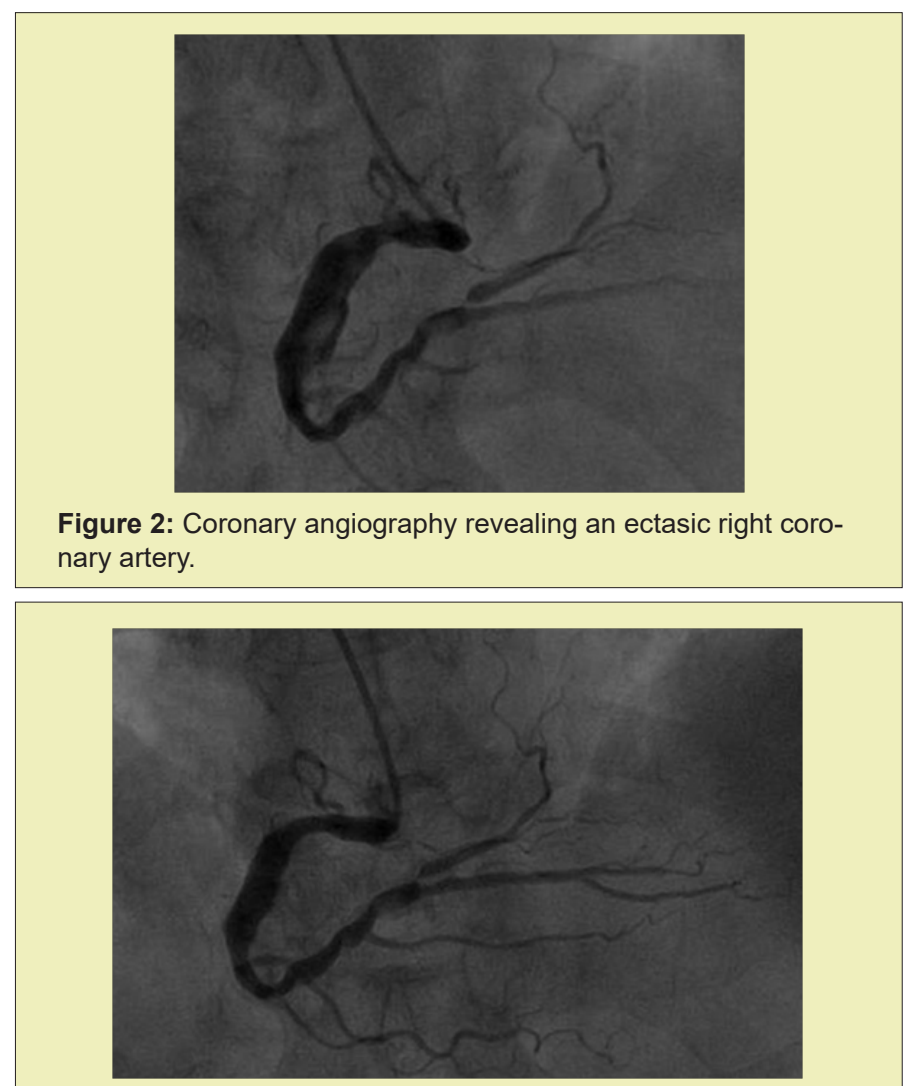

Figure 3: Coronary angiography three months later showing an improvement in the intra mural hematoma.

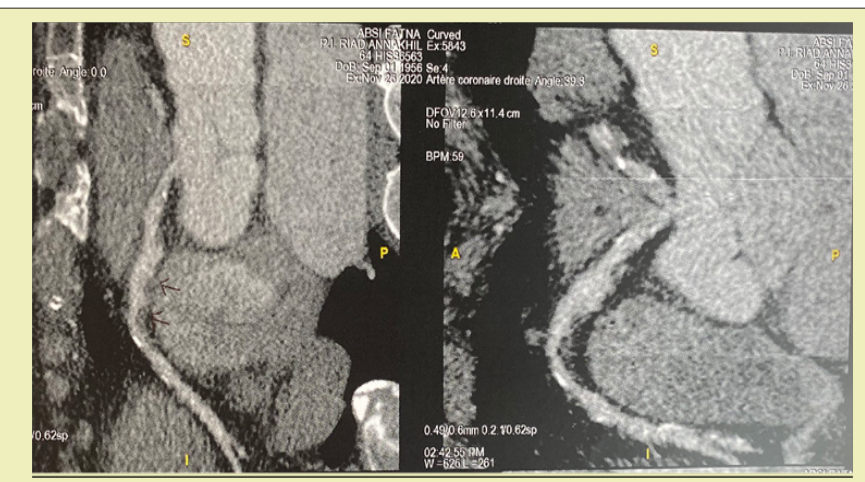

Figure 4: Coronary computed tomography images three months after admission showed an improvement in the intramural hematoma.

\section{Discussion}

Coronary intramural hematomas are specific subtypes of coronary artery dissections. While dissections are pathophysiologically characterized by an initiating intimal tear and propagation of subsequent medial dissection, hematomas are medial dissections with false lumen formation and absence of intimal tear. ${ }^{7}$ In the diagnosis, coronary angiography is not sufficient to identify the cause of stenosis, coronary dissection, or atherosclerotic plaque. Intracoronary imaging techniques such as IVUS and OCT can provide much more detailed information about the morphologic features of the coronary artery, including entry tear, flap, IMH, and associated thrombus. $^{8}$

The proper management of coronary intramural hematomas is currently un--established, but there is a broad agreement on optimal treatment strategy depending on the site of hematoma, number of affected vessels, the reduction in coronary blood flow and hemodynamic state. ${ }^{9}$ Information regarding the long--term prognosis after isolated spontaneous coronary hematomas is extremely sparse due to the limited number of cases reported. However, the prognosis after SCAD is well described and probably transferable. PCI--treatment has been documented with a high rate of complication and procedural failure, and in spite of better long--term survival in comparison with typical ACS--patients, rates of later major adverse events have been found to be high. ${ }^{10}$

\section{Conclusion}

A spontaneous intramural hematoma is one of the possible causes of acute coronary syndrome. intracoronary imaging techniques such as intravascular ultrasound (IVUS) and optical coherence tomography (OCT) can provide detailed morphological infor--mation on coronary lesions, which is useful for an accurate diagnosis and the selection of an appropriate treatment strategy.

\section{Acknowledgement}

None.

\section{Funding}

None.

\section{Conflicts of interest}

Author declares that there is no conflict of interest.

\section{References}

1. Hanratty CG, McKeown PP, 0 `Keeffe DB. Coronary stenting in the setting of sponta-neous coronary artery dissection. Int J Cardiol.1998;67:197-199.

2. DeMaio Jr SJ, Kinsella SH, Silverman ME. Clinicalcourse and long-term prognosis of spontaneous coronary artery dissection. Am J Cardiol. 1989;64:471-474.

3. Raja Y, Trevelyan J, Doshi SN. Intramural hematoma presenting as acute coronary syndrome: the importance of intravascular ultrasound. Cardiol J. 2012;19:323-325.

4. Ikegami R, Tsuchida K, Oda H. Acute myocardial infarction caused by spontaneous coronary intramural hematoma. J Invasive Cardiol. 2012;24:692-693.

5. Poon K, Incani A, Small A, et al. Drug eluting stents trapping intramural hema-tomain spontaneous coronary artery dissection and healing pattern at six months: optical coherence tomography findings. Cardiovasc Revasc Med. 2013;14:183-186. 
6. Gowda RM, Sacchi TJ, Khan IA. Clinical perspectives of the primary spontaneous cor-onary artery dissection. Int J Cardiol. 2005;105:334336.

7. Maehara A, Mintz GS, CastagnaMT,et al. Intravascu-lar assessment of spontaneous coronary artery dissection. Am J Cardiol. 2002;89:466-468.

8. Alfonso F, Paulo M, Gonzalo N, et al. Diagnosis of spontaneous coronary artery dissection by optical coherence tomography. J Am Coll Cardiol. 2012:59:1073-1079.
9. Antonsen L, Thayssen P, Jensen LO. Large coronary intramural hematomas: a case series and focused literature review. Cardiovascular Revasc Med. 2015;16(2):116-23.

10. Tweet MS, Hayes SN, Pitta SR, et al. Clinical features, management, and prognosis of spontaneous coronary artery dissection. Circulation. 2012;126:579-588. 